\title{
Unilateral Maxillary Sinus Aplasia with Ipsilateral Rhinolith: A Rare Anomaly with a Rare Association
}

\author{
${ }^{1}$ Rachna K Tiwari (Nautiyal), ${ }^{2}$ Vaishali S Sangole, ${ }^{3}$ Suman P Rao \\ ${ }^{4}$ Kalpana R Kumar, ${ }^{5}$ Sujata A Gawai, ${ }^{5}$ Divya A George \\ ${ }^{1}$ Senior Resident, Department of ENT, MGM Medical College and Hospital, Navi Mumbai, Maharashtra, India \\ ${ }^{2}$ Lecturer, Department of ENT, MGM Medical College, Navi Mumbai, Maharashtra, India \\ ${ }^{3}$ Professor and Head, Department of ENT, MGM Medical College, Navi Mumbai, Maharashtra, India \\ ${ }^{4}$ P rofessor, Department of ENT, MGM Medical College, Navi Mumbai, Maharashtra, India \\ ${ }^{5}$ Resident, Department of ENT, MGM Medical College, Navi Mumbai, Maharashtra, India
}

Correspondence: Rachna K Tiwari (Nautiyal), Senior Resident, Department of ENT, B-406, Yash Complex, Sector 8 Plot No 21, Kalamboli, Navi Mumbai-410218, Maharashtra, India

\section{ABSTRACT}

Unilateral maxillary sinus aplasia is one of the rare anomalies of maxillary sinus. Few cases have been reported. Ipsilateral rhinolith with unilateral maxillary sinus aplasia is also a very rare association and no case has been documented till date. We report a case of a 21-year-old female with left maxillary sinus aplasia and ipsilateral rhinolith. Rest of the sinuses being normal.

Keywords: Aplasia, Endoscopy, Maxillary sinus.

\section{INTRODUCTION}

Unilateral maxillary sinus aplasia is an uncommon condition that can be diagnosed as chronic sinusitis or neoplasm by the otolaryngologist. ${ }^{1-3}$ Itis one of the causes of radiologically opaque maxillary antrum. ${ }^{4} \mathrm{~W}$ e report a clinical case of this rare entity associated with ipsilateral rhinolith confirmed by CT scan. This extremely rare abnormal ity should be kept in mind to prevent misdiagnosis and possible complications during endoscopic sinus surgery. A short literature review is also presented in this respect.

\section{CASE REPORT}

A 21-year-old female presented with history of left nasal obstruction and foul smelling discharge of 8 months duration. She had no history of recurrent sinusitis, nasal or sinus surgery, trauma, nasal foreign body, tumor, irradiation and no history suggestive of any local bone disease as Paget's disease, osteopetrosis or fibrous dysplasia. Examination revealed normal mental status with anterior rhinoscopy showing yellowish gray crust medial and superior to left inferior turbinate. M iddle turbinate was not visible on left side. Right inferior turbinate was normal and middle turbinate was bullous. Paranasal sinuses examination was normal. There was no evidence of craniofacial anomaly. Tests for blood dyscrasias were negative as were those for thyroid function. Diagnostic nasal endoscopy showed yellowish gray crust medial and superior to left inferior turbinate. Foreign body although suspected was not seen in the left nasal cavity in this diagnostic view. Right middle turbinate was bullous.

Plain CT scan paranasal sinuses (coronal section slices $2 \mathrm{~mm}$ ) revealed absence of left maxillary sinus, uncinate process and infundibulum with enlargement of left nasal fossa, rest of the sinuses being normal. Concha bullosa on right side with slight deviation of septum to left was also seen (Fig. 1).

Functional endoscopic sinus surgery was planned for her, which revealed grayish white material with gritty sensation

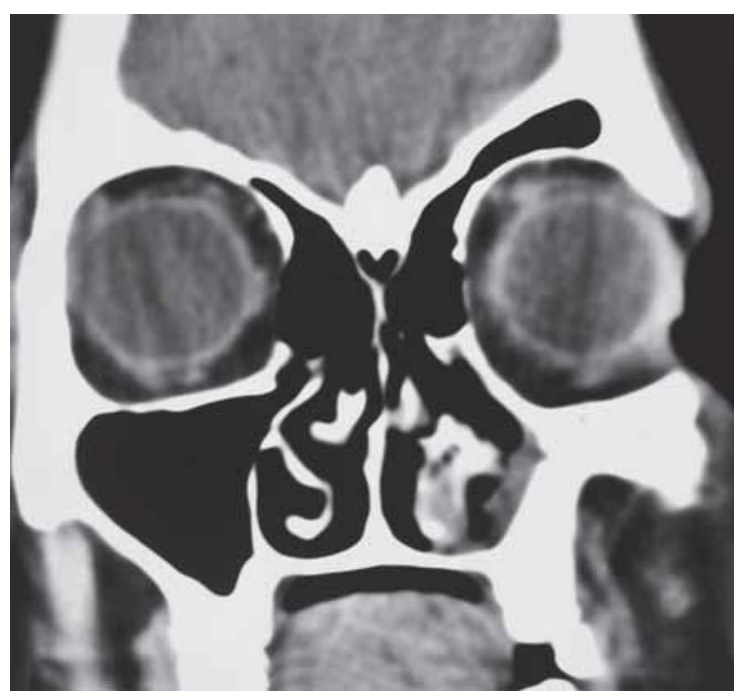

Fig. 1: CT scan of P NS plain coronal section showing absence of maxillary sinus, uncinate process and infundibulum with enlargement of nasal fossa filled with soft tissue of heterogenous opacity on left side 
after displacing theyellowish white crust present medial and superior to left inferior turbinate. Endogenous rhinolith was diagnosed. Uncinate process and maxillary sinus ostia were absent on the left side. R hinolith was removed. Right nasal cavity showed concha bullosa which was excised. Rest all sinuses were normal. Patient was given antibiotic course for 2 weeks and called for follow-up after a week. Patient improved symptomatically. B oth thenasal cavities werewell patent. Follow-up nasal endoscopy was done. No debris, discharge or any residual rhinolith was seen.

\section{DISCUSSION}

$M$ axillary sinus is the first of the paranasal sinuses to develop in the human fetus and present at birth. During the third month of fetal life, the uncinate process projects medially from the ethmoid bone, forming a groove between it and the lateral nasal wall- the infundibulum. This is the site for maxillary sinus cell or bud. The cell enlarges over time and the maxillary sinus becomes radiographically seen by 4 to 5 months. By the age of 12 , pneumatization extends to the orbital wall laterally and to the level of the nasal cavity inferiorly. As permanent dentition occurs, the inferior level of the sinus descends 4 to $5 \mathrm{~mm}$ below this level. ${ }^{5}$

M axillary sinus aplasia is similar to maxillary sinus hypoplasia (MSH) type III in the classification described by B olger et al. M SH type III is characterized by an absent or very hypoplastic uncinate process and profound sinus hypoplasia or aplasia. The sinus is represented by only a shallow cleft in the lateral nasal wall. He found the prevalence of unilateral hypoplastic maxillary sinus to be $10.4 \%$ on coronal CT scans. ${ }^{1} \mathrm{~T}$ o differentiate maxillary sinus aplasia from hypoplasia, thin $(2 \mathrm{~mm}$ ) slices of CT scan are essential. Thin slices also help in better identification of the

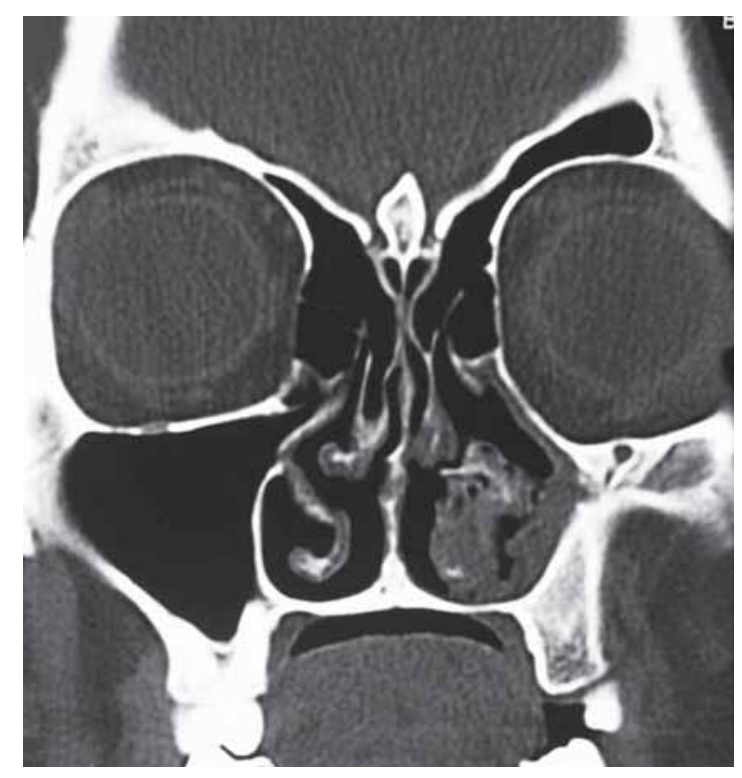

Fig. 2: CT scan of PNS plain coronal section showing well-developed uncinate process on right side while absent uncinate process on left side uncinate process abnormality (Fig. 2), thereby helping the endoscopic sinus surgeon to modify the approach to the osteomeatal complex. In our patient, maxillary sinus, uncinate process as well as infundibulum were absent on left side as evident on nasal endoscopy and CT scan paranasal sinuses ( $2 \mathrm{~mm}$ coronal cuts). So ours is a case of unilateral maxillary sinus aplasia. M axillary sinus aplasia is a very rare congenital anomaly. Only a few cases have been reported in the English literature. 6,7

$M$ axillary sinus aplasia is one of the several causes of radiologically opaque maxillary antrum. U nilateral opacified maxillary antrum on plain films may be due to mucosal thickening in infectious diseases, neoplasia, atelectasis of the sinus and severe hypoplasia or aplasia of the sinus. ${ }^{5,8}$ A telectasis of maxillary sinus resul ts from chronic sinusitis. $\mathrm{M}$ axillary bone loss, collapsed antrum, inward bowing of antral walls and secondary changes in the facial skeleton like enophtalmos are the characteristic features. ${ }^{8} \mathrm{~A}$ II these features represent an acquired disorder rather than a congenital anomaly. Computed tomography (CT) scanning and endoscopic examination are necessary diagnostic tools to detect the underlying abnormality. In our patient, none of the above-mentioned-associated features were present, signifying maxillary sinus aplasia being a congenital anomaly.

Hall GW was one of the first group of authors to propose that the developmental failure of the sinus is an end result of an intrauterine insult. ${ }^{9} \mathrm{H}$ ow ever, sinus infections can also impair normal development of the sinus in the first year of life. Tsue et al reported relation of bilateral maxillary sinus aplasia and bilateral sinonasal papillomatosis. ${ }^{6}$ Human papilloma virus infection can affect the development of paranasal sinuses by teratogenic effect during the intrauterine period. A rrest in development of the maxillary sinus has been related in children with recurrent or chronic rhinosinusitis. The underdevelopment or aplasia of paranasal sinuses is a rare phenomenon that refers mainly to the frontal (12\%) and secondarily to the maxillary sinuses (5-6\%). These are found associated with syndromes of craniosynostosis, osteodysplasia as well as in cases of Down's syndrome (hypoplasia of the frontal sinus). ${ }^{10}$ Eggesbo et $a^{11}$ reported the developmental anomalies of paranasal sinuses in cystic fibrosis patients by comparing them with noncystic fibrosis controls with inflammatory sinusitis. Concomitant aplasia of maxillary and sphenoid sinuses and maxillary sinus aplasia with developmental insufficiency of all paranasal sinuses have been reported. ${ }^{12}$ However, unilateral maxillary sinus aplasia with concomitant existence of a rhinolith and normal rest of the paranasal sinuses has not been reported previously in English literature. This extremely rare anomaly should be kept in mind to prevent misdiagnosis and complications during endoscopic sinus surgery. Though the pathogenesis of rhinolith remains unclear; a number of factors have been proposed like entry and impaction of a foreign body in the nasal cavity, acute and chronic inflammation, obstruction 
and stagnation of nasal secretions, and precipitation of mineral salts. In our case, since there was no history of nasal foreign body and our patient was a mentally sound female, the type of rhinolith is concluded to be endogenous.

With improvement in the diagnostic technique as CT scan, rare cases of maxillary sinus aplasia can be diagnosed at the first sitting. Hence, determining the anatomical variations of maxillary sinus and accompanying structures in patients planned for endoscopic sinus surgery will significantly help with providing surgical orientation and preventing possible complications.

\section{REFERENCES}

1. Bolger WE, Woodruff WW, M orehead J, Parsons DS. $M$ axillary sinus hypoplasia: Classification and description of associated uncinate process hypoplasia. Otolaryngol Head Neck Surg 1990;103:759-65.

2. Kapoor PK, Kumar BN, Watson SD. Maxillary sinus hypoplasia. J Laryngol Otol 2002;116:135-37.

3. Sirikci A, Bayazit $Y$, Gumusburun E, B ayram M, K anlikana M . A new approach to the classification of maxillary sinus hypoplasia with relevant clinical complications. Surg Radiol A nat 2000;22:243-47.

4. Whyte A, Chapeikin G. O paque maxillary antrum: A pictorial review. A ustralas Radiol 2005;49:203-13.

5. Graney DO, Rice DH. A natomy. Otolaryn Head and Neck Surgery. In: Cummings CV (Ed). M osby: St Louis 1993; 901-06.

6. Tsue TT, Bailet JW, Barlow DW, Makielski KH. Bilateral sinonasal papillomas in aplastic maxillary sinuses. Am J Otolaryngol 1997;18:263-68.

7. Clerico DM , Grabo DJ. M axillary sinus aplasia. Ann O tol R hinol Laryngol 1997;106:799-800.

8. Antonelli PJ, Duvall III AJ, Teitelbaum SL. Maxillary sinus atelectasis. Ann Otol Rhinol Laryngol 1992;101:977-81.

9. Hall GW. Embryology and abnormal anatomy of the maxillary sinus. N orthwest M ed 1969;68:1010-11.

10. A nderhuber $W, W$ eiglein $A$, W olf $G$. Nasal cavities and paranasal sinuses in newborns and children. Acta A nat 1992;144:120-26.

11. Eggesbo HB, Sovik S, Dolvik S, Eiklid K, Kolmannskog F. Proposal of a CT scoring system of the paranasal sinuses in diagnosing cystic fibrosis. Eur Radiol 2003;13:1451-60.

12. A ydinlioglu A, Erdem S. M axillary and sphenoid sinus aplasia in Turkish individuals: A retrospective review using computed tomography. Clin A nat 2004;17:618-22.

\section{E ditorial Inputs}

\section{Unilateral Maxillary Sinus Aplasia with Ipsilateral Rhinolith: A Rare Anomaly with a Rare Association}

\begin{abstract}
${ }^{1}$ Bachi T Hathiram, ${ }^{2}$ Vicky S Khattar Mumbai, Maharashtra, India Mumbai, Maharashtra, India

This is truly a rare association which has been reported by the authors. It has numerous surgical implications, the most important being the risk of orbital penetration during endoscopic surgery.
\end{abstract}

${ }^{1}$ Professor and Head, Department of ENT and Head and Neck Surgery, Topiwala National Medical College and BYL Nair Charitable Hospital

${ }^{2}$ Assistant Professor, Department of ENT and Head and Neck Surgery, Topiwala National Medical College and BYL Nair Charitable Hospital

\section{Silent Sinus Syndrome}

A $n$ increasingly common entity which has been described well in the recent literature, the 'silent sinus syndrome' is also known as the the 'imploding antrum' or 'maxillary sinus atelectasis.' Although there is still some confusion regarding these entities, they probably mean the same condition. It is an acquired pathology, hypothesized to be due to an under-aeration or blockage at the level of the osteomeatal complex, resulting in an under-aeration of the maxillary sinus, and resultant inward collapse of its walls. This may result in enophthalmos, hypoglobus, visual disturbances, and cosmetic implications in the long run, with involvement of most walls of the maxillary sinus. Although there may be an underlying 'silent' or 'asymptomatic' chronic sinus pathology, it usually requires an acute episode/trigger of inflammation to manifest. The walls of the maxillary sinus may often be thinned and attenuated, or otherwise normal in thickness. The most commonly involved wall is the orbital floor. This syndrome has also been reported to have an association with ipsilateral proptosis, and it is important to understand this association as a developmental variation without clinical significance, to prevent any undue invasive investigations, and further even surgical exploration of the orbit.

\section{Acquired Hypoplasia/Aplasia}

A nother cause of volumetric decrease in the size of the maxillary sinus could be a fungus ball. It is a known fact that fungus balls are common on the maxillary sinuses, and are usually accompanied by a thickening or hyperostosis of the sinus walls. This may many times result in the decrease in the volume of contained air of the sinus. Presented below is the CT scan of one such patient who had a history of trauma 


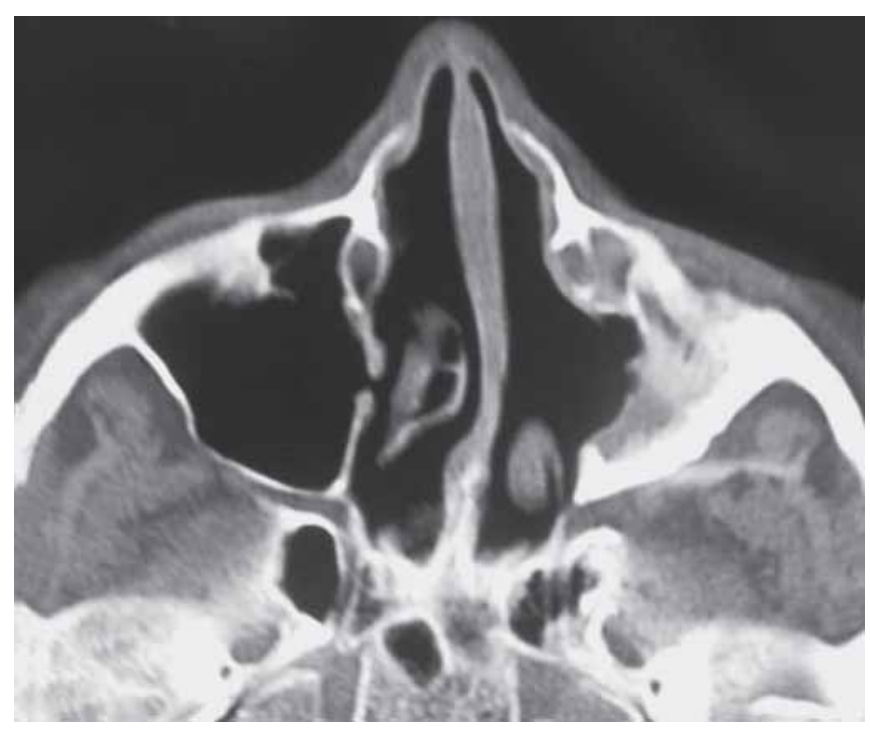

Fig. 1

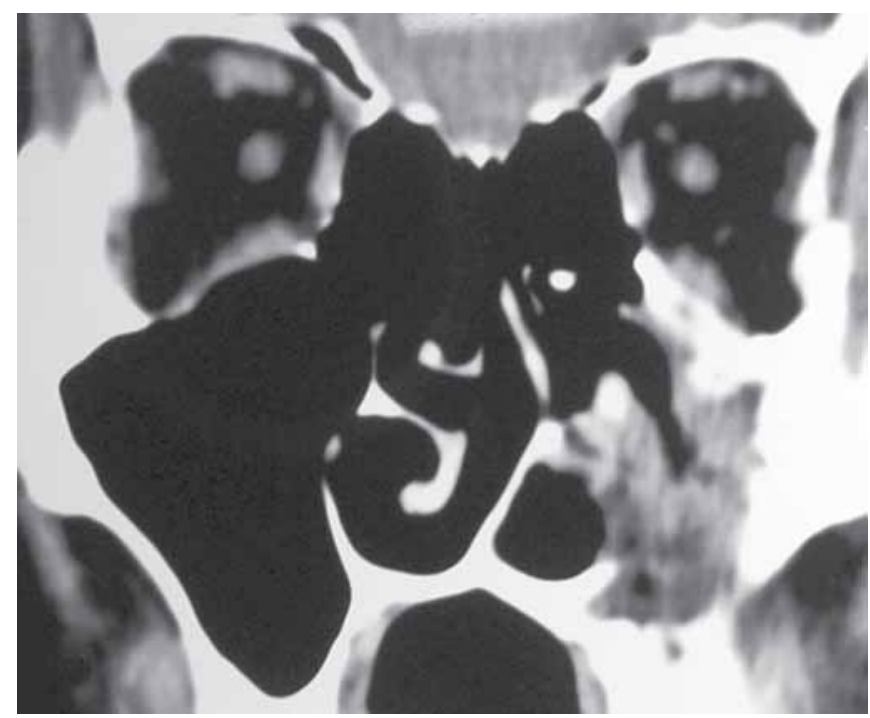

Fig. 2

to the face, followed by a maxillary fracture. An external metallic plating was done to stabilize the same, but had to be removed as it got infected, and the culture of the removed plate revealed an Aspergillus sp. infection. The CT scan showed thickened walls of the maxillary sinus, a marked decrease in lumen, and debris within. When compared with the CT scan image shown in the article "U nilateral M axillary Sinus A plasia with I psilateral R hinolith: A Rare A nomaly with a Rare A ssociation" by Tiwari and colleagues, it can be seen that both the scans show thickening of the maxillary sinus walls. However, the external maxillary sinus contour is mainly unaltered in cases of fungal balls, and the sinus decreases volumetrically from ' within.' The maxillary sinus contour may even be expanded in long-standing cases of fungus balls. In cases of congenital maxillary sinus aplasia however, the external contour is greatly altered.
Plain axial CT scan of the paranasal sinuses showing a post-traumatic left maxillary sinus fungus ball. Note the thickening of the walls, with maintenance of external contour.

Coronal plain CT scan of the same patient. In fact, the fungal debris seen in this image can be easily mistaken for a rhinolith and vice versa (vide the author's CT scan).

\section{Developmental Aplasia}

There have been reports of large concha bullosae being the cause of maxillary sinus hypoplasia, and in many cases, atleast a co-existence of the two has been reported. The eventual outcome being a maxillary sinus atelectasis. This association would, however, be easily evident on a CT scan.

\section{DISCUSSION}

We may thus be encountered with a variety of scenarios.

1. Firstly, a congenital maxillary aplasia, with loss of sinus volume, and loss of external contour. The walls may be thickened. It may lead to chronic ipsilateral underaeration, and resultant sinus pathology, or nasal pathology such as a rhinoloth (as has been reported).

2. There may be a silent sinus, with loss of volume, as well as loss of external contour secondary to indrawing of sinus walls. There is usually loss of sinus wall thickness, most commonly occurring in the roof of maxillary sinus. This may al so be due to a chronic underaeration superimposed by an 'acute episode' which precipitates the entire process.

3. Maxillary sinus fungus balls showing thickened walls, loss of lumen, but with an intact external contour mostly, with occasional external expansion, but usually never any indrawing.

4. A 'developmental' aplasia, in association with a large concha bullosa. The cause-effect relationship of the two can be hypothesized.

\section{BIBLIOGRAPHY}

1. Bierny JP, Dryden R. Orbital Enlargement secondary to paranasal sinus hypoplasia. Am J Roentgenol May 1977;128:850-52.

2. Cobb AR, M urthy R, Cousin GC, El-Rasheed A, TomaA, Uddin $J, M$ anisali $M$. Silent sinus syndrome. Br J O ral M axillofac Surg 2011 Nov 1.

3. deShazo RD, O'Brien M, Chapin K, et al. Criteria for the diagnosis of sinus mycetoma. J Allergy Clin I mmunol 1997;99: 475-85.

4. L und VJ, Lloyd G, Savy L, Howard D. Fungal rhinosinusitis. J ournal of Laryngology and Otology 2000;114:76-80.

5. Plaza G, Ferrando J, M artel J, Toledano A, de los Santos G. $M$ axillary sinus hypoplasia. A cta Otorhinolaryngol Esp M ar 2001;52(2):122-28.

6. Rose GE, Sandy C, Hallberg L, Moseley I. Clinical and radiologic characteristics of the imploding antrum, or "silent sinus," syndrome. O phthalmology A pr 2003;110(4):811-18. 